\title{
GP-Fuzzy Soft Groups
}

\author{
Canan AKIN*
}

Department of Mathematics, Faculty of Arts and Science, Giresun University, 28200, Giresun, Turkey.

Geliş / Received: 22/11/2018, Kabul / Accepted: 11/07/2019

\begin{abstract}
In this paper, the definition of a new concept which is called GP-fuzzy soft subgroup is introduced and some basic properties of the families of $G P$-fuzzy soft subgroups are examined.
\end{abstract}

Keywords: Groups, (Fuzzy) soft set, $U P$-fuzzy soft subset, $G P$-fuzzy soft subgroup

\section{GP-Bulanık Esnek Gruplar}

Öz

Bu makalede, $G P$-bulanık esnek altgrup olarak adlandırılan yeni bir kavram tanıtılmış ve $G P$-bulanık esnek altgrupların ailelerinin bazı temel özellikleri incelenmiştir.

Anahtar Kelimeler: Gruplar, (Bulanık) esnek kümeler, $U P$-bulanık esnek alt küme, GP-bulanık esnek altgrup

\section{Introduction}

The theory of soft sets originally is proposed by Molodtsov (1999) as a mathematical method to deal with uncertainties. In the sequel Maji et al. (2003) present some certain soft binary operations and some basic definitions on soft sets. Ali et al. (2009) discuss some results which are given by Maji et al and they give some new notions. Some potential of the soft sets such as the extension to some certain areas attract the attention of the researchers. Maji et al. (2001) combine the theory of soft set with the theory fuzzy set which was initiated by Zadeh in 1965. Their unified concept is referred as a fuzzy soft set. Yang et al. (2007) study on the notion of fuzzy soft sets. As a subsequent research, Ahmad and Kharal (2009) improve the studies on the fuzzy soft sets and define arbitrary fuzzy soft operations. The extension of fuzzy soft sets to algebraic structures has being interest since its introduction in 1999. Initially, Aygünoğlu and Aygün (2009) define the notion of fuzzy soft groups.

Akın and Karakaya (2018) have proposed a new concept which is called $U P$-fuzzy soft set recently. In their study they introduce the notion of $S P$-fuzzy soft subsemigroup. In this paper, we investigate the notion of $U P$-fuzzy soft set for groups. 


\section{Preliminaries}

A function $f$ from a nonempty set $A$ to the unit interval $[0,1]$ is called a fuzzy subset of $A$ (Zadeh, 1965). $F(A)$ denotes the set of all of the fuzzy subsets of $A$. Let $f, g$ be fuzzy subsets of $A$, then $f \subseteq g$ means that $f(a) \leq$ $g(a)$ for all $a \in A$. For any $\alpha \in[0,1]$, the set $f_{\alpha}=\{a \in A \mid f(a) \geq \alpha\}$ is called the $\alpha$-level set of $f$. Let $\left\{f_{i} \mid i \in \Lambda\right\}$ be a family of the fuzzy subsets of $A$ and $x \in A$. Then $\wedge$ and $\vee$ operations are defined by:

$$
\begin{aligned}
& \left(\hat{i}_{i \in \Lambda}^{\wedge} f_{i}\right)(x)=\hat{N}_{i \in \Lambda} f_{i}(x), \\
& \left(\vee_{i \in \Lambda}^{\vee} f_{i}\right)(x)=\vee_{i \in \Lambda} f_{i}(x),
\end{aligned}
$$

Let $B$ be a subset of $A$, then $\chi_{B}$ denote the characteristic function of $B$ and it is defined by

$$
\chi_{B}(x)= \begin{cases}1, & x \in B \\ 0, & x \notin B .\end{cases}
$$

Let $A, B$ be nonempty sets, $\mu \in F(A), v \in$ $F(B)$ and $f: A \rightarrow B$ be a function. Then $f(\mu) \in F(B)$ is defined by $f(\mu)(b)=$ $\mathrm{V}_{f(a)=b} \mu(a)$ for all $b \in B$ and $f^{-1}(v) \in$ $F(A)$ is defined by $f^{-1}(v)=v(f)$ (Klir and Yuan, 1995).

\subsection{Fuzzy Subgroups}

Let $G$ be group and $f, g \in F(G)$. Then the product operation of $f$ and $g$ is denoted by $f \cdot g$ and defined by $(f \cdot g)(x)=$ $\vee_{x=a b} f(a) \wedge g(b)$ for all $x \in G . f^{-1} \in F(G)$ is defined by $f^{-1}(x)=f\left(x^{-1}\right)$ for all $x \in G$. If, for all $x, y \in G, f(x) \wedge f(y) \leq f(x y)$ and $f(x) \leq f\left(x^{-1}\right)$, then $f$ is called a fuzzy subgroup of $G$.If a fuzzy subgroup $f$ of $G$ satisfies the condition $f(x y)=f(y x)$ for all $x, y \in G$, then it is called a normal fuzzy subgroup of $G . f$ is a normal fuzzy subgroup of $G$ if and only if $f(x y)=f(y x)$ for all $x, y \in G$. (Das,1981; Mordeson and Nair, 2001; Rosenfeld, 1971)

\subsection{Soft Sets}

Some known and useful definitions and notations on soft sets are given in the following.

Definition 2.1. Let $U$ be an initial universal set and $P$ be a set of parameters. Then the power set of $U$ is denoted by $P(U)$. Let $A$ be a subset of $P$. Then a pair $(F, A)$ is called a soft set over $U$ where $F$ is a mapping given by $F: A \rightarrow P(U)$ (Molodtsov, 1999). The pair $(U, P)$ denotes the collection of all soft sets on $U$ with the attributes from $P$ and $(U, P)$ is called a soft class (Kharal and Ahmad, 2011). In this paper, we consider a soft class $(G, P)$ with a group $G$ as the initial universal set.

Definition 2.2. Let $(F, A)$ and $(G, B)$ be two soft sets over $G,(F, A)$ is called a soft subset of $(G, B)$, denoted by $(F, A) \subseteq(G, B)$, if (i) $A \subseteq B, \quad(i i) F(x) \subseteq G(x)$ for each $x \in A$ (Molodtsov, 1999).

Definition 2.3. Let $\left\{\left(F_{i}, A_{i}\right) \mid i \in \Lambda\right\}$ be a family of soft sets in a soft class $(G, P)$. Then

a) The restricted intersection of the family $\left\{\left(F_{i}, A_{i}\right) \mid i \in \Lambda\right\}$, denoted by $\left(\cap_{r}\right)_{i \in \Lambda}\left(F_{i}, A_{i}\right)$, is the soft set $(F, A)$ defined as: $A=\bigcap_{i \in \Lambda} A_{i}, \quad F(x)=$ $\bigcap_{i \in \Lambda} F_{i}(x)(\forall x \in A)$,

b) The extended intersection of the family $\left\{\left(F_{i}, A_{i}\right) \mid i \in \Lambda\right\}$, denoted by $\left(\cap_{e}\right)_{i \in \Lambda}\left(F_{i}, A_{i}\right)$, is the soft set $(F, A)$ defined as: $A=\bigcup_{i \in \Lambda} A_{i}, \quad F(x)=$ $\bigcap_{i \in \Lambda(x)} F_{i}(x) \quad(\forall x \in A) \quad$ where $\Lambda(x)=\left\{i \mid x \in A_{i}\right\}$,

c) The restricted union of the family $\left\{\left(F_{i}, A_{i}\right) \mid i \in \Lambda\right\}, \quad$ denoted by $\left(\cap_{r}\right)_{i \in \Lambda}\left(F_{i}, A_{i}\right)$, is the soft set $(F, A)$ 
defined as: $A=\bigcap_{i \in \Lambda} A_{i}, \quad F(x)=$ $\bigcup_{i \in \Lambda} F_{i}(x)(\forall x \in A)$.

d) The extended union of the family $\left\{\left(F_{i}, A_{i}\right) \mid i \in \Lambda\right\}, \quad$ denoted by $\left(\cap_{e}\right)_{i \in \Lambda}\left(F_{i}, A_{i}\right)$, is the soft set $(F, A)$ defined as: $A=\bigcup_{i \in \Lambda} A_{i}, \quad F(x)=$ $\bigcup_{i \in \Lambda(x)} F_{i}(x)(\forall x \in A)$

e) The $\wedge$-intersection of the family $\left\{\left(F_{i}, A_{i}\right) \mid i \in \Lambda\right\}, \quad$ denoted by $\wedge_{i \in \Lambda}\left(F_{i}, A_{i}\right)$, is the soft set $(F, A)$ defined as: $\quad A=\prod_{i \in \Lambda} A_{i}$, $F\left(\left(x_{i}\right)_{i \in \Lambda}\right)=\bigcap_{i \in \Lambda} F_{i}\left(x_{i}\right)$ $\left(\forall\left(x_{i}\right)_{i \in \Lambda} \in A\right)$,

f) The $\vee$-union of the family $\left\{\left(F_{i}, A_{i}\right) \mid i \in \Lambda\right\}, \quad$ denoted by $\mathrm{\vee}_{i \in \Lambda}\left(F_{i}, A_{i}\right)$, is the soft set $(F, A)$ defined as: $\quad A=\prod_{i \in \Lambda} A_{i}$, $F\left(\left(x_{i}\right)_{i \in \Lambda}\right)=\bigcup_{i \in \Lambda} F_{i}\left(x_{i}\right)$ $\left(\forall\left(x_{i}\right)_{i \in \Lambda} \in A\right)$,

g) The product of the family $\left\{\left(F_{i}, A_{i}\right) \mid i \in \Lambda\right\}, \quad$ denoted by $\prod_{i \in \Lambda}\left(F_{i}, A_{i}\right)$, is the soft set $(F, A)$ defined as: $\quad A=\prod_{i \in \Lambda} A_{i}$, $F\left(\left(x_{i}\right)_{i \in \Lambda}\right)=\prod_{i \in \Lambda} F_{i}\left(x_{i}\right)$

$\left(\forall\left(x_{i}\right)_{i \in \Lambda} \in A\right)$.

(Ali et al., 2009; Çelik et al., 2011; Feng et al., 2008; Kazanc1 et al., 2010; Maji et al., 2003; Pei and Miao, 2005).

\subsection{Fuzzy Soft Sets}

In the following definitions we give some useful and known concepts of fuzzy soft sets.

Definition 2.4. Let $U$ be an initial universal set and $P$ be a set of parameters. A pair $(f, E)$ is called a fuzzy soft set over $U$, where $f$ is a mapping given by $f: E \rightarrow F(U)$ (Maji et al., 2001). The pair $(\widetilde{U, P})$ denotes the collection of all fuzzy soft sets on $U$ with the attributes from $P$ and it is called a fuzzy soft class (Kharal and Ahmad, 2011). In this paper, we consider a fuzzy soft class $(\overline{G, P})$ with a group $G$ as the initial universal set.

Definition 2.5. Let $(f, E)$ be a fuzzy soft set over $U$. For each $\alpha \in[0,1]$, the set $(f, E)_{\alpha}=$ $\left(f_{\alpha}, E\right)$ is called an $\alpha$-level set of $(f, E)$, where $f_{\alpha}(a)=\{x \in G \mid f(a)(x) \geq \alpha\}$ for each $a \in E$. Obviously, $(f, E)_{\alpha}$ is a soft set over $G$ (Aygünoğlu and Aygün, 2009).

Definition 2.6. Let $(f, E)$ and $(g, H)$ be two fuzzy soft sets over $G,(f, E)$ is called a fuzzy soft subset of $(g, H)$, denoted by $(f, E) \subseteq(g, H)$, if (i) $E \subseteq H$, (ii) for each $a \in E, f(a) \leq g(a)$ (Maji et al., 2001).

Definition 2.7. Let $\left\{\left(f_{i}, E_{i}\right) \mid i \in \Lambda\right\}$ be a family of fuzzy soft sets in a fuzzy soft class $(\widetilde{G, P})$. Then

a) The restricted intersection of the family $\left\{\left(f_{i}, E_{i}\right) \mid i \in \Lambda\right\}$, denoted by $\widetilde{\cap}_{i \in \Lambda}^{r}\left(f_{i}, E_{i}\right)$, is a fuzzy soft set $(f, E)$, $E=\bigcap_{i \in \Lambda} E_{i}$ and for all $x \in E$, $f(x)=\Lambda_{i \in \Lambda} f_{i}(x)$.

b) The extended intersection of the family $\left\{\left(f_{i}, E_{i}\right) \mid i \in \Lambda\right\}$, denoted by $\widetilde{\cap}_{i \in \Lambda}^{e}\left(f_{i}, E_{i}\right)$, is a fuzzy soft set $(f, E)$, $E=\bigcup_{i \in \Lambda} E_{i}$ and for all $x \in E$, $f(x)=\Lambda_{i \in \Lambda(x)} f_{i}(x)$ where $\Lambda(x)=$ $\left\{i \mid x \in E_{i}\right\}$,

c) The restricted union of the family $\left\{\left(f_{i}, E_{i}\right) \mid i \in \Lambda\right\}, \quad$ denoted by $\widetilde{\mathrm{U}}_{i \in \Lambda}^{r}\left(f_{i}, E_{i}\right)$, is a fuzzy soft set $(f, E)$, $E=\bigcap_{i \in \Lambda} E_{i}$ and for all $x \in E$, $f(x)=\mathrm{v}_{i \in \Lambda} f_{i}(x)$.

d) The extended union of the family $\left\{\left(f_{i}, E_{i}\right) \mid i \in \Lambda\right\}, \quad$ denoted by $\widetilde{U}_{i \in \Lambda}^{e}\left(f_{i}, E_{i}\right)$, is a fuzzy soft set $(f, E)$, $E=\bigcup_{i \in \Lambda} E_{i}$ and for all $x \in E$, $f(x)=\vee_{i \in \Lambda(x)} f_{i}(x)$.

e) The fuzzy $\wedge$-intersection of the family $\left\{\left(f_{i}, E_{i}\right) \mid i \in \Lambda\right\}, \quad$ denoted by 
$\widetilde{\Lambda}_{\mathrm{i} \in \Lambda}\left(f_{i}, E_{i}\right)$, is the soft set $(f, E)$ defined as: $E=\prod_{i \in \Lambda} E_{i}$, $f\left(\left(x_{i}\right)_{i \in \Lambda}\right)=\Lambda_{i \in \Lambda} f_{i}\left(x_{i}\right) \quad\left(\forall\left(x_{i}\right)_{i \in \Lambda} \in\right.$ $E)$,

f) The fuzzy $\vee$-union of the family $\left\{\left(f_{i}, E_{i}\right) \mid i \in \Lambda\right\}, \quad$ denoted by $\widetilde{\mathrm{V}}_{i \in \Lambda}\left(f_{i}, E_{i}\right)$, is the soft set $(f, E)$ defined as $E=\prod_{i \in \Lambda} E_{i}$, $f\left(\left(x_{i}\right)_{i \in \Lambda}\right)=\mathrm{V}_{i \in \Lambda} f_{i}\left(x_{i}\right) \quad\left(\forall\left(x_{i}\right)_{i \in \Lambda} \in\right.$ $E)$.

g) The product of the family $\left\{\left(f_{i}, E_{i}\right) \mid i \in \Lambda\right\}, \quad$ denoted by $\widetilde{\prod}_{\mathrm{i} \in \Lambda}\left(f_{i}, E_{i}\right)$, is a fuzzy soft set $(f, E), \quad E=\prod_{i \in \Lambda} E_{i} \quad$ and $f\left(\left(x_{i}\right)_{i \in \Lambda}\right)=\vee \underset{J \text { is finite }}{J \subseteq \Lambda}\left(\wedge_{j \in J} f_{j}\left(x_{j}\right)\right)$.

(Ahmad and Kharal, 2009; Çelik et al., 2013; Maji et al., 2001)

Definition 2.8. Let $\left(f, E_{1}\right),\left(g, E_{2}\right)$ be fuzzy soft sets in a fuzzy soft class $(\overline{G, P})$. Then the fuzzy product of them, denoted by $\left(f, E_{1}\right) \widetilde{x}\left(g, E_{2}\right)$, is the soft set $(h, C)$ defined as $C=E_{2} \times E_{1}, \quad h(a, b)=f(a)$. $g(b)$ for all $a \in E_{1}, b \in E_{2}$ (Çelik et al., 2013).

\subsection{Soft Groups and Fuzzy Soft Groups}

Some known and useful definitions on soft and fuzzy soft groups are shown forth as follows.

Definition 2.9. Let $(F, E)$ be a soft set over $G,(F, E)$ is called a soft group over $G$ if and only if $F(a)$ is a subgroup of $G$ for each $a \in$ $E$ (Aktaş and Çağman, 2007; Aslam and Qurashi, 2012; Yin and Liao, 2013).

Definition 2.10. Let $(F, E)$ be a soft group over $G,(F, E)$ is called a normal soft group over $G$ if and only if $F(a)$ is a normal subgroup of $G$ for each $a \in E$ (Aktaş and Çağman, 2007; Aslam and Qurashi, 2012).
Definition 2.11. Let $(f, E)$ be a fuzzy soft set over $G,(f, E)$ is called a fuzzy soft group if and only if $f(a)$ is a fuzzy subgroup over $G$ for each $a \in E$ (Aygünoğlu and Aygün, 2009).

Definition 2.12. Let $(f, E)$ be a fuzzy soft set over $G,(f, E)$ is called a normal fuzzy soft group if and only if $f(a)$ is a normal fuzzy subgroup over $G$ for each $a \in E$ (Aygünoğlu and Aygün, 2009).

Definition 2.13. Let $(f, E)$ be a fuzzy soft group over $G$ and $\alpha, \in[0,1]$. Then $(f, E)$ is said to be a $\alpha$-identity fuzzy soft group over $G$ if, for all $a \in E, x \in G, f(a)(x)=$ $\{\alpha$, if $x=e$ $\{0$, otherwise (Aygünoğlu and Aygün, 2009).

Definition 2.14. Let $\left(U_{1}, P_{1}\right),\left(U_{2}, P_{2}\right)$ be soft classes and $\varphi: U_{1} \rightarrow U_{2}, \psi: P_{1} \rightarrow P_{2}$ be functions. Then the pair $(\varphi, \psi)$ is called a fuzzy soft function from $U_{1}$ to $U_{2}$. (Aygünoğlu and Aygün, 2009).

Definition 2.15. Let $(f, A)$ and $(g, B)$ fuzzy soft sets on the classes $\left(U_{1}, P_{1}\right)$ and $\left(U_{2}, P_{2}\right)$, respectively and let $(\varphi, \psi)$ be called a fuzzy soft function from $U_{1}$ to $U_{2}$. Then

a) The image of $(f, A)$ under the soft function $(\varphi, \psi)$, denoted by $(\varphi, \psi)(f, A)$, is the fuzzy soft set on the class $\left(U_{2}, P_{2}\right)$ defined by $(\varphi, \psi)(f, A)=(\varphi(f), \psi(A))$, where $\varphi(f)(b)(y)=$ $\left\{\begin{array}{cl}\bigvee_{\varphi(x)=y} \bigvee_{\psi(a)=b} f(a)(x), & \text { if } \exists x \in \varphi^{-1}(y) \\ 0 & \text { otherwise }\end{array}\right.$ , $\left(\forall b \in \psi(A)\right.$ and $\left.\forall y \in U_{2}\right)$.

b) The pre-image of $(g, B)$ under the fuzzy soft function $(\varphi, \psi)$, denoted by $(\varphi, \psi)^{-1}(g, B)$, is defined by $(\varphi, \psi)^{-1}(g, B)=\left(\varphi^{-1}(g), \psi^{-1}(B)\right)$, 
where

$g(\psi(a))(\varphi(x))$,

$\left.\psi^{-1}(B), \forall x \in U_{1}\right)$

(Aygünoğlu and Aygün, 2009).

Definition 2.16. Let $(\varphi, \psi)$ be a fuzzy soft function from $G$ to $H$. If $\varphi$ is a homomorphism from $G$ to $H$ then $(\varphi, \psi)$ is said to be fuzzy soft homomorphism (Aygünoğlu and Aygün, 2009).

\subsection{UP-Fuzzy Soft Sets}

Akın and Karakaya (2018) propose a fuzzy soft set of a crisp soft set as new concept of a member of the class $(\widetilde{U, P})$. They give the following definitions.

Definition 2.17. Let $(F, A)$ be a soft set in a soft class $(U, P)$ and $(f, E)$ be a fuzzy soft set in the fuzzy soft class $(\widetilde{U, P})$. Then $(f, E)$ is said to be a $U P$-fuzzy soft subset of $(F, A)$, denoted by $(f, E) \subseteq_{U P}(F, A)$, if $E \subseteq A$ and $f(x)$ is a fuzzy subset of $F(x)$ for all $x \in E$.

Definition 2.18. Let $(f, E)$ be a $U P$-fuzzy soft subset of $(F, A) .\left(F_{\alpha}, E\right)$ called $\alpha$-level soft subset of $(f, E)$, where $F_{\alpha}: E \rightarrow P(U)$ is defined by $F_{\alpha}(x)=$ $\{a \in F(x) \mid f(x)(a) \geq \alpha\}=f(x)_{\alpha}$ for all $x \in E$.

Definition 2.19. Let $(f, E)$ be a $U P$-fuzzy soft subset of $(F, A)$. Then the $U P$-fuzzy soft subset $(f, E)^{c}:=\left(f^{c}, E\right)$ of $(F, A)$ is called the complement of $(f, E)$, where for any $x \in$ $E, \quad f^{c}(x): F(x) \rightarrow[0,1]$ is defined by $f^{c}(x)(a)=1-f(x)(a)$ for all $a \in F(x)$.

Definition 2.20. Let $S$ be a semigroup and let $(F, A)$ be a soft subsemigroup in a soft class $(S, P)$ and $(f, E)$ be a fuzzy soft set in the fuzzy soft class $\overline{(S, P)} .(f, E)$ is called a $S P$ -

\section{GP-Fuzzy Soft Groups}

In this section we give a definition of a new concept which is called GP-fuzzy soft group, where $G$ is a group. Then we investigate some properties of GP-fuzzy soft group. Throughout this section $G, H, N$ will be considered as groups.

Definition 3.1. Let $(F, A)$ be a soft group in a soft class $(G, P)$ and $(f, E)$ be a fuzzy soft set in the fuzzy soft class $(\overline{G, P}) .(f, E)$ is called a $G P$-fuzzy soft group of $(F, A)$ if $E \subseteq$ $A$ and $f(x)$ is a fuzzy subgroup of $F(x)$ for all $x \in E$.

\section{Example 3.2.}

a) Let $G$ be the group $\left(\mathbb{Z}_{6},+\right)$ and $P=$ $\left\{x_{1}, x_{2}, x_{3}\right\}$. Let $A=\left\{x_{1}, x_{2}\right\}$ and $F\left(x_{1}\right)=\{\overline{0}\}$ and $F\left(x_{2}\right)=\{\overline{0}, \overline{2}, \overline{4}\}$. Let $E=\left\{x_{2}\right\}$ and $(f, E)$ be defined by $f\left(x_{2}\right)(\bar{a})=\left\{\begin{array}{l}1, \text { if } \bar{a}=\overline{0}, \\ 0, \text { otherwise }\end{array}\right.$ for all $\bar{a} \in F\left(x_{2}\right)$. Then $(f, E)$ is a $G P$ fuzzy soft group of $(F, A)$.

b) Let $G$ be the general linear group of matrices in $\mathbb{R}$ of type $2 \times 2$ and $P=$ $\left\{x_{1}, x_{2}, x_{3}\right\}$. Let $A=\left\{x_{1}, x_{2}\right\}$ and $F\left(x_{1}\right)=\left\{\left[\delta_{i j}\right]\right\}$ and $F\left(x_{2}\right)$ be the special linear group of matrices in $\mathbb{R}$ of type $2 \times 2$, where $\left[\delta_{i j}\right]$ is unit matrix. Let $E=\left\{x_{2}\right\}$ and $(f, E)$ be defined by

$f\left(x_{2}\right)\left(\left[a_{i j}\right]\right)=\left\{\begin{array}{l}1, \text { if } a_{i j}=\delta_{i j}, \\ 0, \text { otherwise },\end{array}\right.$, $\left(\forall\left[a_{i j}\right] \in F\left(x_{2}\right)\right)$. Then $(f, E)$ is a $G P$-fuzzy soft group of $(F, A)$. 
Remark. Let $(f, E)$ be a fuzzy soft set in the fuzzy soft class $(\overline{G, P})$. If $(f, E)$ is a fuzzy soft group of $G$ then it is a $G P$-fuzzy soft group of $(F, A)$. Therefore the class of $G P$ fuzzy soft groups of a soft group covers the class of fuzzy soft groups and the inverse of this is not true in general. For instance, in Example $3.2(\mathrm{~b})$, the function $f\left(x_{2}\right)$ is not defined for the matrices which have the determinant different from 1 .

Theorem 3.3. Let $(F, A)$ be a soft group over $G$ and $(f, E)$ be a $G P$-fuzzy soft subset of $(F, A)$. Then $\left(F_{\alpha}, E\right)$, if $f(x)_{\alpha} \neq \varnothing$ for all $x \in$ $E$ and for any $\alpha \in(0,1]$, is a soft group over $G$ for all $\alpha \in[0,1]$ if and only if $(f, E)$ is $G P$-fuzzy soft subgroup of $(F, A)$.

Proof. Suppose that $\left(F_{\alpha}, E\right)$ is a soft group for all $\alpha \in[0,1]$. Let $x \in E$ and $\alpha$ := $f(x)(a) \wedge f(x)(b)$ for any $a, b \in F(x)$. So $f(x)(a) \geq \alpha$ and $f(x)(b) \geq \alpha$. Thus $a, b \in$ $F_{\alpha}(x) . a b^{-1} \in F_{\alpha}(x)$ since $F_{\alpha}(x)$ is a subgroup of $G$ for all $x \in E$. So $f(x)\left(a b^{-1}\right) \geq \alpha, \quad$ i.e, $\quad f(x)\left(a b^{-1}\right) \geq$ $f(x)(a) \wedge f(x)(b)$. Hence $f(x): F(x) \rightarrow$ $[0,1]$ is fuzzy subgroup for all $x \in E$. Therefore $(f, E)$ is a $G P$-fuzzy soft group of $(F, A)$. On the contrary, let $a, b \in F_{\alpha}(x)$ for any $\alpha \in[0,1]$. Hence $f(x)(a) \geq \alpha$ and $f(x)(b) \geq \alpha$. Thus $f(x)(a) \wedge f(x)(b) \geq \alpha$. Thus $f(x)\left(a b^{-1}\right) \geq \alpha$ since $f(x)$ is a fuzzy subgroup of $F(x)$ for all $x \in E$. So $a b^{-1} \in$ $F_{\alpha}(x)$. Therefore $\left(F_{\alpha}, E\right)$ is a soft group for all $\alpha \in[0,1]$.

Theorem 3.4. Let $\left(f_{i}, E_{i}\right)$ be GP-fuzzy soft group of $\left(F_{i}, A_{i}\right)$ for all $i \in \Lambda$. Then
a) $\widetilde{\cap}_{i \in \Lambda}^{r}\left(f_{i}, E_{i}\right)$ is $G P$-fuzzy soft group of $\left(\cap_{r}\right)_{i \in \Lambda}\left(F_{i}, A_{i}\right)$.
b) $\widetilde{\cap}_{i \in \Lambda}^{e}\left(f_{i}, E_{i}\right)$ is $G P$-fuzzy soft group of $\left(\cap_{e}\right)_{i \in \Lambda}\left(F_{i}, A_{i}\right)$.
c) $\widetilde{\Pi}_{i \in \Lambda}^{r}\left(f_{i}, E_{i}\right)$ is $G P$-fuzzy soft group of $\left(\bigcap_{e}\right)_{i \in \Lambda}\left(F_{i}, A_{i}\right)$.
d) $\widetilde{\prod}_{i \in \Lambda}\left(f_{i}, E_{i}\right)$ is a GP-fuzzy soft group of $\prod_{i \in \Lambda}\left(F_{i}, A_{i}\right)$.

Proof.

a) Let $\widetilde{\Pi}_{i \in \Lambda}^{r}\left(f_{i}, E_{i}\right)=(f, E) \quad$ and $\left(\cap_{r}\right)_{i \in \Lambda}\left(F_{i}, A_{i}\right)=(F, A)$. Clearly, $E=\bigcap_{i \in \Lambda} E_{i} \subseteq \bigcap_{i \in \Lambda} A_{i}=A$. Let $a, b \in F(x)$ for any $x \in E$. $f(x)\left(a b^{-1}\right)=\left(\wedge_{i \in \Lambda} f_{i}(x)\right)\left(a b^{-1}\right)=$ $\Lambda_{i \in \Lambda}\left(f_{i}(x)\left(a b^{-1}\right)\right) \geq$ $\wedge_{i \in \Lambda}\left(f_{i}(x)(a) \wedge f_{i}(x)(b)\right)=$ $\wedge_{i \in \Lambda}\left(f_{i}(x)(a)\right) \wedge \Lambda_{i \in \Lambda}\left(f_{i}(x)(b)\right)=$ $f(x)(a) \wedge f(x)(b)$ for all $a, b \in$ $F(x)$ since $F(x)=\bigcap_{i \in \Lambda} F_{i}(x)$. We obtain that $f(x)$ is fuzzy subgroup of $F(x)$ for all $x \in E$. Thus $\widetilde{\cap}_{i \in \Lambda}^{r}\left(f_{i}, E_{i}\right)$ is a $G P$-fuzzy soft group of $\left(\cap_{r}\right)_{i \in \Lambda}\left(F_{i}, A_{i}\right)$.

b) Let $\widetilde{\Pi}_{i \in \Lambda}^{e}\left(f_{i}, E_{i}\right)=(f, E) \quad$ and $\left(\bigcap_{e}\right)_{i \in \Lambda}\left(F_{i}, A_{i}\right)=(F, A)$. Clearly, $E=\bigcup_{i \in \Lambda} E_{i} \subseteq \bigcup_{i \in \Lambda} A_{i}=A$. Let $i \in$ $\Lambda$ be arbitrary and constant, and $J=$ $\left\{j \in \Lambda \mid x \in E_{j}, i \neq j\right\}$, and $x \in E$. If $x \in E_{i}$, then there are two cases: $x \in$ $E_{i} \backslash \bigcup_{i \neq j} E_{j} \quad$ or $\quad J \neq \emptyset$. If $x \in$ $E_{i} \backslash \bigcup_{i \neq j} E_{j}$, then $F(x)=F_{i}(x)$ and $f(x)=f_{i}(x)$, and since $f(x)$ is a fuzzy subgroup of $F(x)$, then $(f, E)$ is $G P$-fuzzy soft group of $(F, A)$. If $J \neq \varnothing$, then $F(x) \subseteq\left(\bigcap_{j \in J} F_{j}(x)\right) \cap$ $F_{i}(x)$ and $f(x)=\left(\bigwedge_{j \in J} f_{j}(x)\right) \wedge$ $f_{i}(x)$. Hence $(f, E)$ is GP-fuzzy soft group of $(F, A)$ by similar way in (i).

c) Let $x \notin E_{i}$. Then $F(x) \subseteq \bigcap_{j \in J} F_{j}(x)$ and $f(x)=\Lambda_{j \in J} f_{j}(x)$. Hence $(f, E)$ is $G P$-fuzzy soft group of $(F, A)$ by similar way in (i). 
d) Let $\widetilde{\cap}_{i \in \Lambda}^{r}\left(f_{i}, E_{i}\right)=(f, E) \quad$ and $\left(\cap_{e}\right)_{i \in \Lambda}\left(F_{i}, A_{i}\right)=(F, A)$. Then $E=$ $\bigcap_{i \in \Lambda} E_{i} \subseteq \bigcap_{i \in \Lambda} A_{i} \subseteq \bigcup_{i \in \Lambda} A_{i}=A$.

Let $x \in E$. Thus $x \in \bigcap_{i \in \Lambda} A_{i}$. Hence $f(x)=\Lambda_{i \in \Lambda} f_{i}(x) \quad$ and $\quad F(x)=$ $\bigcap_{i \in \Lambda} F_{i}(x)$. Since $\Lambda_{i \in \Lambda} f_{i}(x)$ is a fuzzy subgroup of $\bigcap_{i \in \Lambda} F_{i}(x),(f, E)$ is a $G P$-fuzzy soft subgruop of $(F, A)$.

e) Let $\widetilde{\prod}_{i \in \Lambda}\left(f_{i}, E_{i}\right)=(f, E) \quad$ and $\prod_{i \in \Lambda}\left(F_{i}, A_{i}\right)=(F, A)$. Clearly, $E=$ $\prod_{i \in \Lambda} E_{i} \subseteq \prod_{i \in \Lambda} A_{i}=A$. Let $a, b \in$ $F\left(\left(x_{i}\right)_{i \in \Lambda}\right)$ for any $\left(x_{i}\right)_{i \in \Lambda} \in E$. Then

$$
\begin{aligned}
& f\left(\left(x_{i}\right)_{i \in \Lambda}\right)(a) \wedge f\left(\left(x_{i}\right)_{i \in \Lambda}\right)(b)=\left(\underset{J \text { is finite }}{\vee \subseteq \Lambda}\left(\wedge_{i \in J}^{\wedge} f_{i}\left(x_{i}\right)\right)\right)(a) \wedge\left(\underset{J \subseteq \Lambda}{\vee \subseteq \Lambda} \underset{J \text { is finite }}{\vee}\left(\wedge_{i \in J} f_{i}\left(x_{i}\right)\right)\right)(b) \\
& =\underset{J \subseteq}{\vee} \underset{J \text { is finite }}{\vee}\left(\wedge_{i \in J}^{\wedge} f_{i}\left(x_{i}\right)(a)\right) \wedge \underset{J \text { is finite }}{\vee} \underset{i \in J}{\vee}\left(\wedge_{i \in J} f_{i}\left(x_{i}\right)(b)\right) \\
& =\underset{\substack{J \subseteq \Lambda \\
J \text { is finite }}}{\vee}\left(\wedge_{i \in J}\left(f_{i}\left(x_{i}\right)(a) \wedge f_{i}\left(x_{i}\right)(b)\right)\right) \\
& \leq \underset{J \text { is finite }}{\vee}\left(\wedge_{i \in J}^{\wedge}\left(f_{i}\left(x_{i}\right)\left(a b^{-1}\right)\right)\right)=\underset{\substack{J \subseteq \Lambda \\
J \text { is finite }}}{\vee}\left(\wedge_{i \in J}^{\wedge} f_{i}\left(x_{i}\right)\right)\left(a b^{-1}\right) \\
& =f\left(\left(x_{i}\right)_{i \in \Lambda}\right)\left(a b^{-1}\right) \text {. }
\end{aligned}
$$

Thus $\widetilde{\prod}_{i \in \Lambda}\left(f_{i}, E_{i}\right)$ is a $G P$-fuzzy soft group of $\prod_{i \in \Lambda}\left(F_{i}, A_{i}\right)$ since $f\left(\left(x_{i}\right)_{i \in \Lambda}\right)$ is a fuzzy subgroup of $F\left(\left(x_{i}\right)_{i \in \Lambda}\right)$.

\section{Definition 3.5.}

a) Let $(f, E)$ be a fuzzy soft group over $G$ and $\alpha, \beta \in[0,1], \beta \leq \alpha$. Then $(f, E)$ is said to be a $(\alpha, \beta)$-identity fuzzy soft group over $G$ if, for all $a \in$ $E, x \in G, f(a)(x)=\left\{\begin{array}{l}\alpha, \text { if } x=e ; \\ \beta, \text { otherwise. }\end{array}\right.$

b) Let $(f, E)$ be a $G P$-fuzzy soft group of $(F, A)$ and $\alpha, \beta \in[0,1], \beta \leq \alpha$. Then $(f, E)$ is said to be a $(\alpha, \beta)$-identity fuzzy soft group of $(F, A)$ if, for all $a \in E, x \in F(a)$, $f(a)(x)=$ $\{\alpha$, if $x=e$;

$\{$, otherwise.

Theorem 3.6. Let $\varphi$ be a homomorphism from $F(G)$ to $F(H)$ and $(f, E)$ is a fuzzy soft group over $G$ defined by $f(a)(x)=$ $\left\{\begin{array}{l}\alpha, \text { if } x \in \operatorname{Ker} \varphi ; \\ \beta, \text { otherwise. }\end{array}\right.$
Then $(\varphi(f), E)$ is a $(\alpha, \beta)$-identity fuzzy soft group over $H$.

Proof. $\varphi(f)(a)\left(e_{H}\right)=\mathrm{V}_{\varphi(x)=e_{H}} f(a)(x)=$ $\mathrm{V}_{x \in \operatorname{Ker} \varphi} f(a)(x)=\alpha$.

If $y \neq e_{H}$ then $\varphi(f)(a)(y)=\beta$. Therefore $(\varphi(f), E)$ is a $(\alpha, \beta)$-identity fuzzy soft group over $H$.

Theorem 3.7. Let $\varphi$ be a homomorphism from $F(G)$ to $F(H)$ and $(f, E)$ be a $G P$-fuzzy soft group of $(F, A)$ defined by $f(a)(x)=$ $\left\{\begin{array}{l}\alpha, \text { if } x \in \operatorname{Ker} \varphi ; \\ \beta, \text { otherwise. }\end{array}\right.$ $a \in E$. Then $(\varphi(f), E)$ is a $(\alpha, \beta)$-identity fuzzy soft group of $(\varphi(F), A)$.

Proof. It is straightforward.

Theorem 3.8. Let $(f, E)$ be a $G P_{1}$-fuzzy soft group of $(F, A)$. If $(\varphi, \psi)$ is a fuzzy soft homomorphism then $(\varphi, \psi)(f, E)$ is a $H_{P_{2}}$ fuzzy soft group of $(\varphi(F), \psi(A))$. 
Proof. Let $y_{1}, y_{2} \in \varphi(F)(b)$ for any $b \in$ such that $\varphi\left(x_{1}\right)=y_{1}$ and $\varphi\left(x_{2}\right)=y_{2}$. Then $\psi(E)$. Suppose that there exist $x_{1}, x_{2} \in G$

$$
\begin{aligned}
\varphi(f)(b)\left(y_{1} y_{2}^{-1}\right) & \geq \bigvee_{\varphi\left(x_{1}\right)=y_{1}} \bigvee_{\psi(a)=b} f(a)\left(x_{1}\right) \wedge \bigvee_{\varphi\left(x_{2}\right)=y_{2}} \bigvee_{\psi(a)=b} f(a)\left(x_{2}\right) \\
= & \varphi(f)(b)\left(y_{1}\right) \wedge \varphi(f)(b)\left(y_{2}\right)
\end{aligned}
$$

since the inequality

$$
\begin{aligned}
\varphi(f)(b)\left(y_{1} y_{2}^{-1}\right) & =\bigvee_{\varphi(x)=y_{1} y_{2}^{-1}} \bigvee_{\psi(a)=b} f(a)(x) \geq \bigvee_{\psi(a)=b} f(a)\left(x_{1} x_{2}^{-1}\right) \\
& \geq \bigvee_{\psi(a)=b} f(a)\left(x_{1}\right) \wedge f(a)\left(x_{2}\right)=\bigvee_{\psi(a)=b} f(a)\left(x_{1}\right) \wedge \bigvee_{\psi(a)=b} f(a)\left(x_{2}\right)
\end{aligned}
$$

is satisfied for each $x_{1}, x_{2} \in G$ such that homomorphism then $(\varphi, \psi)^{-1}(g, B)$ is a $\varphi\left(x_{1}\right)=y_{1} \quad$ and $\quad \varphi\left(x_{2}\right)=y_{2}$. Thus $G P_{1}$-fuzzy $\quad$ soft group of $(\varphi, \psi)(f, E)$ is a $H P_{2}$-fuzzy soft group of $\left(\varphi^{-1}(T(\psi)), \psi^{-1}(K)\right)$. $(\varphi(F), \psi(A))$.

Theorem 3.9. Let $(g, B)$ be a $H P_{2}$-fuzzy soft $\psi^{-1}(B)$. Then group of $(T, K)$. If $(\varphi, \psi)$ is a fuzzy soft

$$
\begin{gathered}
\varphi^{-1}(g)(a)\left(x_{1} x_{2}^{-1}\right)=g(\psi(a))\left(\varphi\left(x_{1} x_{2}^{-1}\right)\right)=g(\psi(a))\left(\varphi\left(x_{1}\right) \varphi\left(x_{2}^{-1}\right)\right) \\
\geq g(\psi(a))\left(\varphi\left(x_{1}\right)\right) \wedge g(\psi(a))\left(\varphi\left(x_{2}\right)\right)=\varphi^{-1}(g)(a)\left(x_{1}\right) \wedge \varphi^{-1}(g)(a)\left(x_{2}\right) .
\end{gathered}
$$

$\operatorname{Thus}(\varphi, \psi)^{-1}(g, B)$ is a $G P_{1}$-fuzzy soft group of $\left(\varphi^{-1}(T(\psi)), \psi^{-1}(K)\right)$.

Definition 3.10. Let $(f, E)$ be $G P$-fuzzy soft group of $(F, A) .(f, E)$ is called a $G P$-normal fuzzy soft group of $(F, A)$ if and only if $f(a)$ is a normal fuzzy subgroup over $F(a)$ for each $a \in E$.

Example 3.11. In Example $3.2(a),(f, E)$ is a $G P$-normal fuzzy soft group of $(F, A)$. In Example $3.2(\mathrm{~b}),(f, E)$ is not a $G P$-normal fuzzy soft group of $(F, A)$.

Theorem 3.12. Let $(F, A)$ be a soft group over $G$ and $(f, E)$ be a $G P$-fuzzy soft subset of $(F, A)$. a) If $\left(F_{\alpha}, E\right)$ is a normal soft group for all $\alpha \in[0,1]$ then $(f, E)$ is a $G P$ normal fuzzy soft group of $(F, A)$.

b) Let $(F, A)$ be a normal soft subgroup over $G$. If $(f, E)$ is a $G P$ normal fuzzy soft group of $(F, A)$ then $\left(F_{\alpha}, E\right)$, if $f(x)_{\alpha} \neq \emptyset$ for all $x \in E$ and for any $\alpha \in(0,1]$, is a normal soft group of $(F, A)$ for all $\alpha \in[0,1]$.

Proof.

a) Let $a, b \in F(x)$ for all $x \in E$ and $\alpha:=f(x)(b)$. Then $b \in F_{\alpha}(x)$. Hence $a b a^{-1} \in F_{\alpha}(x)$ since $\left(F_{\alpha}, E\right)$ is a soft normal subgroup for all $\alpha \in$ $[0,1]$. So $f(x)\left(a b a^{-1}\right) \geq \alpha$. Thus $f(x)\left(a b a^{-1}\right) \geq f(x)(b)$. Therefore 
$(f, E)$ is a $G P$-normal fuzzy soft subgroup of $(F, A)$.

b) Let $\alpha \in[0,1] ; a \in F_{\alpha}(x)$ and $b \in$ $F(x)$. Thus $f(x)\left(b a b^{-1}\right) \geq$ $f(x)(a) \geq \alpha$ since $(f, E)$ is a $G P$ normal fuzzy soft group of $(F, A)$. Hence $b a b^{-1} \in F_{\alpha}(x)$. Therefore $\left(F_{\alpha}, E\right)$ is a normal soft group of $(F, A)$ for all $\alpha \in[0,1]$ from Theorem 3.3.

Theorem 3.13. Let $\left(f_{i}, E_{i}\right)$ be $G P$-normal fuzzy soft group of $\left(F_{i}, A_{i}\right)$ for all $i \in \Lambda$. Then

a) $\widetilde{\Pi}_{i \in \Lambda}^{r}\left(f_{i}, E_{i}\right)$ is $G P$-normal fuzzy soft group of $\left(\bigcap_{r}\right)_{i \in \Lambda}\left(F_{i}, A_{i}\right)$.

b) $\widetilde{\cap}_{i \in \Lambda}^{e}\left(f_{i}, E_{i}\right)$ is $G P$-normal fuzzy soft group of $\left(\bigcap_{e}\right)_{i \in \Lambda}\left(F_{i}, A_{i}\right)$.

c) $\widetilde{\Pi}_{i \in \Lambda}^{r}\left(f_{i}, E_{i}\right)$ is $G P$-normal fuzzy soft group of $\left(\cap_{e}\right)_{i \in \Lambda}\left(F_{i}, A_{i}\right)$.

d) $\widetilde{\prod}_{i \in \Lambda}\left(f_{i}, E_{i}\right)$ is a $G P$-normal fuzzy soft group of $\prod_{i \in \Lambda}\left(F_{i}, A_{i}\right)$.
Proof.

a) Let $\widetilde{\Pi}_{i \in \Lambda}^{r}\left(f_{i}, E_{i}\right)=(f, E) \quad$ and $\left(\cap_{r}\right)_{i \in \Lambda}\left(F_{i}, A_{i}\right)=(F, A) . \quad$ Clearly $E=\bigcap_{i \in \Lambda} E_{i} \subseteq \bigcap_{i \in \Lambda} A_{i}=A$. Let $a, b \in F(x) \quad$ for any $\quad x \in E$. $f(x)(a b)=\left(\wedge_{i \in \Lambda} f_{i}(x)\right)(a b)=$ $\Lambda_{i \in \Lambda}\left(f_{i}(x)(a b)\right)=$ $\wedge_{i \in \Lambda}\left(f_{i}(x)(b a)\right)=$ $\wedge_{i \in \Lambda}\left(f_{i}(x)(b a)\right)=$ $\left(\wedge_{i \in \Lambda} f_{i}(x)\right)(b a)=f(x)(b a)$ for all $a, b \in F(x) \quad$ since $\quad F(x)=$ $\bigcap_{i \in \Lambda} F_{i}(x)$. We obtain that $f(x)$ is fuzzy normal subgroup of $F(x)$ for all $x \in E$ with Theorem 3.4. Thus $\widetilde{\Pi}_{i \in \Lambda}^{r}\left(f_{i}, E_{i}\right)$ is a $G P$-normal fuzzy soft group of $\left(\cap_{r}\right)_{i \in \Lambda}\left(F_{i}, A_{i}\right)$.

b) It is straightforward.

c) It is straightforward.

d) Let $\widetilde{\prod}_{i \in \Lambda}\left(f_{i}, E_{i}\right)=(f, E) \quad$ and $\prod_{i \in \Lambda}\left(F_{i}, A_{i}\right)=(F, A)$. Clearly $E=$ $\prod_{i \in \Lambda} E_{i} \subseteq \prod_{i \in \Lambda} A_{i}=A$. Let $a, b \in$ $F\left(\left(x_{i}\right)_{i \in \Lambda}\right)$ for any $\left(x_{i}\right)_{i \in \Lambda} \in E$. Then

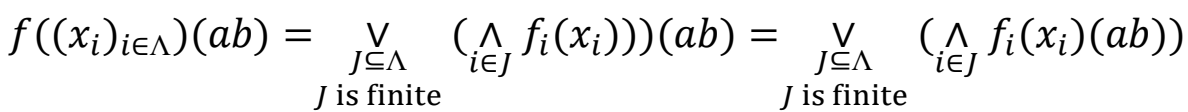

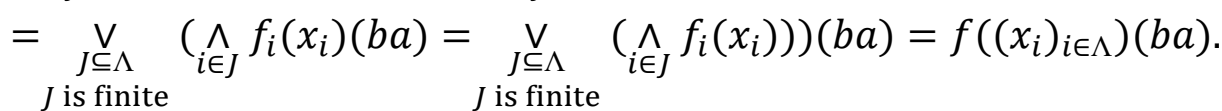

Thus $\widetilde{\prod}_{i \in \Lambda}\left(f_{i}, E_{i}\right)$ is a $G P$-normal fuzzy soft group of $\prod_{i \in \Lambda}\left(F_{i}, A_{i}\right)$ with Theorem 3.5.

Definition 3.14. Let $\left(f, E_{1}\right),\left(g, E_{2}\right)$ be fuzzy soft sets in a fuzzy soft class $(\overline{G, P})$. Then the fuzzy intersection product of them, denoted by $\left(f, E_{1}\right) \cdot\left(g, E_{2}\right)$, is the soft set $(h, C)$ defined as $C=E_{1} \cap E_{2}, h(x)=f(x) \cdot g(x)$ for all $x \in E_{1} \cap E_{2}$.
Definition 3.15. Let $\left(f, E_{1}\right)$ be $G P$-fuzzy soft group of $\left(F, A_{1}\right)$ and $\left(g, E_{2}\right)$ be $G P$-fuzzy soft group of $\left(G, A_{2}\right)$. Then the fuzzy intersection product of them, denoted by $\left(f, E_{1}\right) \approx\left(g, E_{2}\right)$, is the soft set $(h, C)$ defined as $C=E_{1} \cap E_{2}, h(x)=f(x) \circ g(x)$ for all $x \in E_{1} \cap E_{2}$, where

$$
(f(x) \circ g(x))(s)=\left\{\begin{array}{cc}
\bigvee_{s=p q} f(x)(p) \wedge g(x)(q), \exists p \in F(x), q \in G(x): s=p q \\
0, & \text { otherwise. }
\end{array}\right.
$$


Theorem 3.16. Let $\left(\mathrm{G}, A_{2}\right)$ be a normal soft group over $G$ and let $\left(f, E_{1}\right)$ be $G P$-fuzzy soft group of $\left(F, A_{1}\right)$ and $\left(g, E_{2}\right)$ be $G P$-normal fuzzy soft group of $\left(\mathrm{G}, A_{2}\right)$. Then $\left(f, E_{1}\right) \widetilde{\circ}\left(g, E_{2}\right)$ is a $G P$-fuzzy soft group of $\left(\mathrm{F}, A_{1}\right) \cap\left(\mathrm{G}, A_{2}\right)$.
Proof. Let $\left(f, E_{1}\right) \approx\left(g, E_{2}\right)=(h, C)$ and $\left(\mathrm{F}, A_{1}\right) \cap\left(\mathrm{G}, A_{2}\right)=(H, D)$. Then $C \subseteq D$. Let $a, b \in H(x)$ for any $x \in E_{1} \cap E_{2}$. Thus $h(x)$ is a fuzzy subgroup of $H(x)$ since

$$
\begin{aligned}
& h(x)(a) \wedge h(x)(b)=(f(x) \circ g(x))(a) \wedge(f(x) \circ g(x))(b)=\left(\vee_{a=p t} f(x)(p) \wedge g(x)(t)\right) \wedge \\
& \left(\mathrm{V}_{b=u v} f(x)(u) \wedge g(x)(v)\right)=\mathrm{V}_{a=p t}(f(x)(p) \wedge f(x)(u) \wedge g(x)(t) \wedge g(x)(v)) \leq \\
& V_{\substack{a=p t \\
b^{-1}=v^{-1} u^{-1}}}\left(f(x)\left(p u^{-1}\right) \wedge g(x)\left(t v^{-1}\right)\right)=\vee_{\substack{a=p t \\
b^{-1}=v^{-1} u^{-1}}}\left(f(x)\left(p u^{-1}\right) \wedge g(x)\left(u t v^{-1} u^{-1}\right)\right) \leq \\
& V_{a b^{-1}=p t v^{-1} u^{-1}}\left(f(x)\left(p u^{-1}\right) \wedge g(x)\left(u t v^{-1} u^{-1}\right)\right) .
\end{aligned}
$$

Definition 3.17. Let $\left(f, E_{1}\right)$ be $G P$-fuzzy soft of them, denoted by $\left(f, E_{1}\right) \widetilde{*}\left(g, E_{2}\right)$, is the group of $\left(F, A_{1}\right)$ and $\left(g, E_{2}\right)$ be $G P$-fuzzy soft set $(h, C)$ defined as $C=E_{2} \times E_{1}$, soft group of $\left(G, A_{2}\right)$. Then the fuzzy product $h(a, b)=f(a) * g(b)$ for all $a \in E_{1}, b \in E_{2}$, where

$$
(f(a) * g(b))(s)=\left\{\begin{array}{cc}
\bigvee_{s=p q} f(a)(p) \wedge g(b)(q), \exists p \in F(a), q \in G(b): s=p q \\
0, & \text { otherwise. }
\end{array}\right.
$$

Theorem 3.18. Let $\left(\mathrm{G}, A_{2}\right)$ be a normal soft group over $G$ and let $\left(f, E_{1}\right)$ be $G P$-fuzzy soft group of $\left(F, A_{1}\right)$ and $\left(g, E_{2}\right)$ be $G P$-normal fuzzy soft group of $\left(\mathrm{G}, A_{2}\right)$. Then $\left(f, E_{1}\right) \tilde{*}\left(g, E_{2}\right)$ is a $G P$-fuzzy soft group of $\left(\mathrm{F}, A_{1}\right) \wedge\left(\mathrm{G}, A_{2}\right)$.
Proof. Let $\left(f, E_{1}\right) \tilde{*}\left(g, E_{2}\right)=(h, C)$ and $\left(\mathrm{F}, A_{1}\right) \wedge\left(\mathrm{G}, A_{2}\right)=(H, D)$. Then $C \subseteq D$. Let $a, b \in H(x, y)$ for any $(x, y) \in E_{1} \times E_{2}$. Thus $h(x, y)$ is a fuzzy subgroup of $H(x, y)$ since

$$
\begin{aligned}
& h(x, y)(a) \wedge h(x, y)(b)=(f(x) * g(y))(a) \wedge(f(x) * g(y))(b)=\left(\vee_{a=p t} f(x)(p) \wedge\right. \\
& g(y)(t)) \wedge\left(\mathrm{V}_{b=u v} f(x)(u) \wedge g(y)(v)\right)=\underset{\substack{a=p t \\
b=u v}}{ }(f(x)(p) \wedge f(x)(u) \wedge g(y)(t) \wedge \\
& g(y)(v)) \leq \vee_{\substack{a=p t \\
b^{-1}=v^{-1} u^{-1}}}\left(f(x)\left(p u^{-1}\right) \wedge g(y)\left(t v^{-1}\right)\right)=\bigvee_{b^{-1}=v^{-1} u^{-1}}^{a=p t}\left(f(x)\left(p u^{-1}\right) \wedge\right. \\
& \left.g(y)\left(u t v^{-1} u^{-1}\right)\right) \leq V_{a b^{-1}}=p t v^{-1} u^{-1}\left(f(x)\left(p u^{-1}\right) \wedge g(y)\left(u t v^{-1} u^{-1}\right)\right) .
\end{aligned}
$$

Theorem 3.19. Let $(f, E)$ be a $G P_{1}$-normal fuzzy soft group of $(F, A)$. If $(\varphi, \psi)$ is a fuzzy soft homomorphism then $(\varphi, \psi)(f, E)$ is a $H P_{2}$-normal fuzzy soft group of $(\varphi(F), \psi(A))$.
Proof. Let $y_{1}, y_{2} \in \varphi(F)(b)$ for any $b \in$ $\psi(E)$. Suppose that there exist $x_{1}, x_{2} \in G$ such that $\varphi\left(x_{1}\right)=y_{1}$ and $\varphi\left(x_{2}\right)=y_{2}$. Then

$$
\varphi(f)(b)\left(y_{1} y_{2}\right) \geq \bigvee_{\varphi(x)=y_{2} y_{1}} \bigvee_{\psi(a)=b} f(a)(x)=\varphi(f)(b)\left(y_{2} y_{1}\right)
$$


since the inequality

$$
\varphi(f)(b)\left(y_{1} y_{2}\right)=\bigvee_{\varphi(x)=y_{1} y_{2}} \bigvee_{\psi(a)=b} f(a)(x) \geq \bigvee_{\psi(a)=b} f(a)\left(x_{1} x_{2}\right)=\bigvee_{\psi(a)=b} f(a)\left(x_{2} x_{1}\right)
$$

is satisfied for each $x_{1}, x_{2} \in G$ such that fuzzy soft homomorphism then $\varphi\left(x_{1}\right)=y_{1}$ and $\varphi\left(x_{2}\right)=y_{2}$. Thus $(\varphi, \psi)^{-1}(g, B)$ is a $G P_{1}$-normal fuzzy soft $(\varphi, \psi)(f, E)$ is a $H P_{2}$-normal fuzzy soft group of $\left(\varphi^{-1}(T(\psi)), \psi^{-1}(K)\right)$.

group of $(\varphi(F), \psi(A))$.

Theorem 3.20. Let $(g, B)$ be a $H P_{2}$-normal $\psi^{-1}(B)$. Then fuzzy soft group of $(T, K)$. If $(\varphi, \psi)$ is a

$$
\begin{gathered}
\varphi^{-1}(g)(a)\left(x_{1} x_{2}\right)=g(\psi(a))\left(\varphi\left(x_{1} x_{2}\right)\right)=g(\psi(a))\left(\varphi\left(x_{1}\right) \varphi\left(x_{2}\right)\right)= \\
g(\psi(a))\left(\varphi\left(x_{2}\right) \varphi\left(x_{1}\right)\right)=g(\psi(a))\left(\varphi\left(x_{2} x_{1}\right)\right)=\varphi^{-1}(g)(a)\left(x_{2} x_{1}\right) .
\end{gathered}
$$

$\operatorname{Thus}(\varphi, \psi)^{-1}(g, B)$ is a $G P_{1}$-normal fuzzy soft group of $\left(\varphi^{-1}(T(\psi)), \psi^{-1}(K)\right)$.

\section{References}

Ahmad, B. and Kharal, A. 2009. "On Fuzzy Soft Sets", Advances in Fuzzy Systems, 2009, 586-507.

Akın C. and Karakaya Ü. 2018. "SP-Fuzzy Soft Ideals in Semigroups", Turkish Journal of Mathematics and Computer Science, 10, 22-32.

Aktaş H. and Çağman N. 2007. "Soft Sets and Soft Groups", Information Sciences, 177, 2726-2735.

Ali, M. I., Feng, F., Liu, -X., Min, -W.K. and Shabir, M. 2009. On Some New Operations in Soft Set Theory, Computers and Mathematics with Applications, 57, 15471553.

Aslam M. and Qurashi S. M. 2012. "Some Contributions to Soft Groups", Annals of Fuzzy Mathematics and Informatics, 14, 1, 177-195.
Aygünoğlu, A. and Aygün, H. 2009. "Introduction to Fuzzy Soft Groups", Comput. Math. Appl., 58, 1279-1286.

Das, P. S. 1981. "Fuzzy Groups and Level Subgroups", J. Math. Anal. Appl. 84, 264269.

Çelik, Y., Ekiz, C. and Yamak, S. 2011. "A New View on Soft Rings", Hacettepe Journal of Mathematics and Statistics, 40, 273-286.

Çelik, Y., Ekiz, C. and Yamak, S. 2013. "Applications of Fuzzy Soft Sets in Ring Theory", Annals Fuzzy Mathematics and Informatics, 5, 451-462.

Feng, F., Jun, -Y.B. and Zhao, -X. 2008. "Soft Semirings", Computers and Mathematics with Applications, 56, 26212628.

Kazanc1, O., Y1lmaz, S, . and Yamak, S. 2010. Soft Sets and Soft Bch-algebras, Hacettepe Journal of Mathematics and Statistics, 39, 205-217.

Kharal, A. and Ahmad, B. 2011. Mappings on Soft Classes, New Mathematics and Natural Computation, 7, 471-481. 
Klir, G.J. and Yuan, B. (1995). "Fuzzy Sets and Fuzzy Logic, in: Theory and Applications", Prentice-Hall Inc, New Jersey.

Maji, P.K., Biswas, R. and Roy, A.R. 2003. Soft Set Theory, Computers and Mathematics with Applications, 45, 555-562.

Maji, P.K., Biswas, R. and Roy, A.R. 2001. Fuzzy Soft Sets, J. Fuzzy Math., 9, 589-602.

Molodtsov, D. 1999. Soft Set Theory First Results, Computers and Mathematics with Applications, 37, 19-31.

Mordeson J.N., Nair P.S. (2001). "Fuzzy Abstract Algebra. In: Fuzzy Mathematics". Studies in Fuzziness and Soft Computing, vol 20, Physica, Heidelberg.

Pei, D. and Miao, D. 2005. From Soft Sets to Information Systems, Granular Computing, 2005 IEEE International Conference on (2), 617-621.

Rosenfeld, A. 1971. Fuzzy Groups, J. Math. Anal. Appl., 35, 512-517.

Yang, X., Yu, D., Yang, J. and Wu, C. 2007. Generalization of Soft Set Theory: From Crisp to Fuzzy Case, in Proceedings of the 2nd International Conference of Fuzzy Information and Engineering (ICFIE O^ C,O* 07), 40 of Advances in Soft Computing, 345354.

Yin X. and Liao Z. 2013. Study on Soft Groups. Journal of Computers 8: 960-967.

Zadeh, L. A. 1965. Fuzzy Sets, Inform. and Control 8, 338-353. 